\section{Avaliação do trabalho dos Núcleos Ampliados de Saúde da Família e Atenção Báscia (NASF-AB) por usuários, segundo os atributos da atenção primária}

\author{
Users' assessment of the work by the Expanded \\ Family Health and Basic Healthcare Centers \\ (NASF-AB) according to attributes of primary care \\ Evaluación del trabajo de los Núcleos Ampliados \\ de Salud de la Familia y Atención Básica \\ (NASF-AB) por parte de usuarios, según las \\ características de la atención primaria
}

Diane Costa Moreira 1 José Patrício Bispo Júnior 1 Adriana Alves Nery 2 Jefferson Paixão Cardoso 2

doi: 10.1590/0102-311X00031420

\title{
Resumo
}

O estudo objetivou avaliar o trabalho dos Núcleos Ampliados de Saúde da Família e Atenção Básica (NASF-AB), na perspectiva dos usuários, a partir dos atributos da atenção primária à saúde (APS) abrangente. Trata-se de estudo de avaliação qualitativa do tipo casos múltiplos, desenvolvido em seis municípios do Estado da Bahia, Brasil. Utilizou-se como referencial teórico-metodológico uma matriz avaliativa fundamentada nos atributos de acesso, longitudinalidade e integralidade. A matriz também considerou os pressupostos do Apoio Matricial e as diretrizes da Estratégia Saúde da Família. As fontes de dados e informações foram 44 entrevistas com usuários do NASF-AB e observação das atividades dos núcleos e das rotinas das unidades de saúde. Evidenciou-se que os usuários têm acesso restrito às atividades dos núcleos. A disponibilidade de atendimentos clinicos mostrou-se insuficiente, a acessibilidade foi facilitada pela realização de visitas domiciliares e a aceitabilidade ficou abalada diante das frustrações por expectativas não cumpridas. A longitudinalidade do cuidado não tem sido priorizada no trabalho dos núcleos, com frágil envolvimento dos apoiadores no desenvolvimento do cuidado continuado. As atividades grupais demonstraram potencialidade para formação de vínculo. No atributo da integralidade, foi identificado que a presença dos núcleos contribuiu para o incremento de ações e a resolutividade, no entanto a articulação com a rede de atenção foi inexpressiva. Conclui-se que o trabalho do NASF-AB apresentou restrições para o desenvolvimento da APS abrangente. Os resultados também sugerem potencialidades com capacidade de fortalecer os cuidados primários que são pouco explorados.

\author{
Correspondência \\ J. P. Bispo Júnior \\ Núcleo de Epidemiologia e Saúde Coletiva, Instituto \\ Multidisciplinar de Saúde, Universidade Federal da Bahia. \\ Rua Hormindo Barros 58, Vitória da Conquista, BA \\ 45029-094, Brasil. \\ jpatricio@ufba.br \\ ${ }^{1}$ Instituto Multidisciplinar em Saúde, Universidade Federal da \\ Bahia, Vitória da Conquista, Brasil. \\ 2 Universidade Estadual do Sudoeste da Bahia, Jequié, Brasil.
}




\section{Introdução}

A atenção primária em saúde (APS) constitui-se como conjunto de práticas e ações de atenção ambulatorial de primeiro nível, direcionadas a responder às necessidades de saúde mais comuns, sejam individuais sejam coletivas ${ }^{1}$. No âmbito internacional, há um acirrado debate sobre diferentes modelos de APS, a partir de duas concepções principais polarizadas. A APS abrangente caracteriza-se como um conjunto de serviços integrados a um sistema de saúde de acesso universal e tem prestação de serviços de primeiro contato, longitudinalidade, integralidade e coordenação do cuidado 2 como atributos principais. A segunda vertente, denominada de APS seletiva, corresponde a uma atenção direcionada para grupos populacionais específicos, com pacote limitado de serviços e baixa resolutividade 3 .

No Brasil, o termo empregado pelo movimento sanitário, especialmente nas primeiras décadas após a criação do Sistema Único de Saúde (SUS), foi atenção básica (AB) à saúde. Para Cecílio \& Reis ${ }^{4}$, a preferência por $A B$ pode ser considerada como uma diferenciação ideológica para se contrapor à ideia de APS seletiva.

Concepções díspares fomentam debates entre o uso de APS ou AB. De acordo com Giovanella 5, há imprecisões de ambos os termos, que podem corresponder a políticas muito distintas. A APS pode referir-se à perspectiva abrangente ou constituir-se como seletiva e focalizada. Não obstante, atenção básica pode também aproximar-se da ideia de "serviços básicos de saúde", correspondente a uma cesta de serviços médicos restritos 5 . Neste sentido, no presente trabalho, será adotada a terminologia de APS em sentido abrangente e integral 2, fundamentada nos princípios do SUS, na busca de garantir universalidade, integralidade e equidade dos cuidados primários 4 .

A Estratégia Saúde da Família (ESF) é o principal mecanismo estruturante da APS no país, embora outros formatos de equipes sejam previstos pela Política Nacional de Atenção Básica (PNAB) 6. As ações da ESF, ao longo dos anos, promoveram consideráveis avanços e conquistas, no sentido da ampliação de acesso aos serviços de saúde 3, diminuição da morbimortalidade por causas evitáveis $7 \mathrm{e}$ diminuição das iniquidades em saúde 8 . Não obstante os avanços alcançados, persistem ainda desafios relacionados à integralidade do cuidado 9 , às questões estruturais de financiamento 7 e à integração com a Rede de Atenção à Saúde (RAS) 10, dentre outros.

Diversas estratégias e programas foram pensados para superação dos desafios impostos à ESF, com destaque para a criação, em 2008, dos Núcleos de Apoio à Saúde da Família (NASF), com o propósito de aumento da resolutividade e ampliação do escopo de ações na APS 11. Ao longo dos seus 12 anos de existência, os núcleos passaram por reformulação em sua composição e nomenclatura. A PNAB de 20176 estabeleceu que os núcleos se chamariam Núcleo Ampliado de Saúde da Família e Atenção Básica (NASF-AB). Por sua vez, outra portaria do Ministério da Saúde 12 utiliza a denominação de Núcleo Ampliado de Saúde da Família e Atenção Primária (NASF-AP). Diante da indefinição conceitual, neste artigo, será adotada a denominação NASF.

Estudos sobre a atuação e o desempenho dos núcleos têm revelado importantes contribuições para a APS. Aspectos como a ampliação do escopo de ações 13 e a melhoria da saúde e do bem-estar 14 são evidenciados em diferentes cenários. Por sua vez, dificuldades para o desempenho do trabalho se relacionam à infraestrutura inapropriada 15 , à frágil integração entre as equipes $16 \mathrm{e}$ ao excesso de demanda na atenção básica ${ }^{17}$. Embora esses achados e reflexões contribuam para o aprimoramento do trabalho do NASF e para o fortalecimento da APS, chama a atenção que a maior parte das publicações indica estudos desenvolvidos com profissionais dos núcleos ou das equipes apoiadas.

Poucos estudos contemplam as avaliações dos usuários sobre o trabalho dos apoiadores 14,18. Estudos com usuários e que tomem por referência os atributos da APS para avaliar o trabalho dos núcleos não foram identificados na literatura. De acordo com Fausto et al. 10, a experiência dos usuários é também uma importante dimensão para avaliar o desempenho dos serviços de saúde. Isso é considerado um componente-chave para identificar problemas e dificuldades que afetam a qualidade do cuidado prestado. Nesse sentido, as expectativas e as experiências de quem utiliza os serviços precisam ser consideradas na avaliação e organização do NASF, a fim de se superarem as fragilidades sentidas e avançar no fortalecimento dos cuidados primários.

O presente artigo teve como objetivo avaliar o trabalho do NASF, na perspectiva dos usuários, a partir dos atributos da APS abrangente. 


\section{Métodos}

Estudo de avaliação qualitativa do tipo casos múltiplos, com níveis de análise imbricados ${ }^{19}$, que tem como objeto o trabalho do NASF.

O NASF constitui-se como arranjo organizacional e assistencial inovador no âmbito do SUS e tem suscitado discussões sobre suas finalidades e seu lugar na RAS. De acordo com os documentos oficiais 6,11 e acadêmicos 13,17,20, o NASF situa-se na APS e tem a finalidade de ampliar o leque de serviços e adensar o arsenal tecnológico do primeiro nível de contato. Todavia, outros entendimentos sugerem que os núcleos se configurem como atenção secundária 21,22. Para Tesser 21, as diretrizes ministeriais geram ambiguidades e superposições entre equipes de saúde da família (EqSF) e NASF. Assim, caberia aos núcleos realizar, precipuamente, cuidados especializados relacionados a competências nucleares, e não a atividades coletivas generalistas. De acordo com Vendruscolo et al. 22, o NASF pode se situar no contexto da APS, mas também para além dela, constituindo-se, assim, em zona intermediária entre cuidados primários e atenção secundária. Para os autores, o NASF deve ser identificado como uma nova atenção especializada, coordenada pela APS.

Neste trabalho, considera-se que os núcleos são parte integrante da APS e devem se estruturar e organizar conforme os princípios do cuidado universal, integral e equânime. Desse modo, parte-se do pressuposto de que o trabalho do NASF deve também coadunar-se com os atributos da APS abrangente.

O campo do estudo constituiu-se de seis municípios pertencentes à região de saúde do Sudoeste da Bahia. Os municípios foram selecionados a partir do critério de ter NASF em funcionamento há mais de um ano e de ter assentimento da gestão municipal para realização da pesquisa, sendo eles: Vitória da Conquista, Guanambi, Brumado, Poções, Barra do Choça e Belo Campo. As características dos municípios no ano de realização da pesquisa estão apresentadas na Tabela 1.

Como aparato teórico-metodológico, foi utilizada uma matriz teórica fundamentada nos atributos da APS abrangente 2,25,26, na metodologia do Apoio Matricial 27,28, bem como nas diretrizes do NASF e da ESF 6,11. Essa matriz considerou os seguintes atributos: acesso, longitudinalidade e integralidade. No Quadro 1, são apresentadas as dimensões utilizadas para a análise de cada atributo.

O acesso foi entendido como uso oportuno dos serviços de saúde para alcançar os melhores resultados possíveis, considerando a disponibilidade, a acessibilidade e a aceitabilidade. Nesse sentido, espera-se que os NASF contribuam para eliminação das barreiras geográficas, financeiras, organizacionais e culturais, o que possibilita a utilização dos serviços de acordo com a necessidade de usuários.

$\mathrm{O}$ atributo da longitudinalidade teve como pressuposto a atribuição de responsabilidade longitudinal com indivíduos e comunidades ao longo do tempo e foi composta das seguintes dimensões: continuidade da atenção e vínculo. Para os núcleos ampliados, a longitudinalidade refere-se ao acompanhamento, em articulação com as EqSF, de casos desafiadores, sejam individuais sejam coletivos.

Tabela 1

Caracterização dos municípios e das equipes estudadas. Municípios participantes da pesquisa, Bahia, Brasil, 2017.

\begin{tabular}{|c|c|c|c|c|c|c|}
\hline Município & $\begin{array}{l}\text { População * } \\
\text { (2017) }\end{array}$ & $\begin{array}{c}\text { Taxa de ubanização } \\
\star *(2010)\end{array}$ & $\begin{array}{l}\text { IDHM ** } \\
(2010)\end{array}$ & $\begin{array}{l}\text { Número de EqSF * } \\
\text { (2017) }\end{array}$ & $\begin{array}{l}\text { Cobertura da ESF * } \\
\qquad(2017)\end{array}$ & $\begin{array}{c}\text { Número de NASF * } \\
\text { (2017) }\end{array}$ \\
\hline Barra do Choça & 35.200 & 64,41 & 0,551 & 12 & 100,00 & 1 \\
\hline Belo Campo & 18.459 & 56,36 & 0,575 & 06 & 96,25 & 1 \\
\hline Brumado & 69.022 & 69,86 & 0,656 & 15 & 74,49 & 3 \\
\hline Guanambi & 85.237 & 79,36 & 0,673 & 19 & 75,94 & 2 \\
\hline Poções & 48.655 & 77,53 & 0,604 & 15 & 94,07 & 2 \\
\hline $\begin{array}{l}\text { Vitória da } \\
\text { Conquista }\end{array}$ & 340.199 & 89,53 & 0,678 & 44 & 44,86 & 4 \\
\hline
\end{tabular}

EqSF: equipes de saúde da família; ESF: Estratégia Saúde da Família; IDHM: Índice de Desenvolvimento Humano Municipal;

NASF: Núcleo de Apoio à Saúde da Família

Fontes: * Ministério da Saúde 23; ** Programa das Nações Unidas para o Desenvolvimento 24. 
Quadro 1

Atributos e dimensões de análise da atenção primária à saúde (APS) integral e atuação do Núcleo de Apoio à Saúde da Família (NASF).

\begin{tabular}{|c|c|c|}
\hline Atributo & Dimensão & Descrição da dimensão e relação com o trabalho do NASF \\
\hline \multirow[t]{3}{*}{ Acesso } & Disponibilidade & $\begin{array}{l}\text { Existência de serviços de saúde em local apropriado e no tempo oportuno. Reflete a } \\
\text { possibilidade de contato do usuário com os profissionais de apoio. Refere-se aos tipos de } \\
\text { serviços e ações disponibilizados pelo NASF para situações previstas e imprevistas. }\end{array}$ \\
\hline & Acessibilidade & $\begin{array}{l}\text { Formas de acesso das pessoas aos serviços e ações de saúde diante das condições } \\
\text { geográficas, organizacionais e financeiras. Essas condições referem-se à distância da casa } \\
\text { do usuário aos serviços de saúde, ao tempo percorrido e às formas de organização que } \\
\text { possibilitam às pessoas utilizarem os serviços. Sobre as ações do NASF, a acessibilidade é } \\
\text { determinada pelos mecanismos e fluxos para se ter contato com os profissionais de apoio. }\end{array}$ \\
\hline & Aceitabilidade & $\begin{array}{l}\text { Percepção e satisfação dos usuários em relação aos serviços prestados. Refere-se às } \\
\text { expectativas e impressões dos usuários em relação aos serviços, a como estão organizados } \\
\text { e à postura do profissional diante das necessidades. Sobre o NASF, relaciona-se à aceitação } \\
\text { do usuário às ações ofertadas, bem como ao reconhecimento da importância dos } \\
\text { profissionais do apoio. }\end{array}$ \\
\hline \multirow[t]{2}{*}{ Longitudinalidade } & $\begin{array}{c}\text { Continuidade da } \\
\text { atenção }\end{array}$ & $\begin{array}{l}\text { Refere-se à continuidade da relação entre profissionais/equipes e usuários/famílias } \\
\text { ao longo do tempo, independente da presença ou ausência de doença. A atuação do } \\
\text { NASF deve se desenvolver em conjunto com as EqSF para o estabelecimento do cuidado } \\
\text { continuado. Pressupõe a responsabilização das equipes pelos casos que demandam } \\
\text { atenção especial e continuada, seja por atenção direta, seja pelo apoio prestado à equipe } \\
\text { de referência. }\end{array}$ \\
\hline & Vínculo & $\begin{array}{l}\text { Estabelecimento de relação de confiança entre usuários e serviços/profissionais de } \\
\text { saúde. Os profissionais devem conhecer as necessidades e problemas dos indivíduos e } \\
\text { famílias. Busca o envolvimento do usuário na produção do cuidado. Para o NASF, significa } \\
\text { a responsabilização e o estabelecimento de confiança com os usuários e a promoção da } \\
\text { autonomia dos indivíduos com corresponsabilização sobre os problemas de saúde. }\end{array}$ \\
\hline \multirow[t]{3}{*}{ Integralidade } & $\begin{array}{c}\text { Abrangência de } \\
\text { ações }\end{array}$ & $\begin{array}{l}\text { Reconhecimento pelos profissionais de saúde da amplitude das necessidades dos } \\
\text { indivíduos e comunidades de todas as faixas etárias, nos diversos âmbitos. Refere-se à } \\
\text { atuação do NASF para a ampliação das ações desenvolvidas na atenção primária à saúde, } \\
\text { no que tange à promoção da saúde, prevenção de agravos, cura e reabilitação. A ampliação } \\
\text { das ações pode se dar por meio do apoio às equipes de saúde da família ou de atividades } \\
\text { desenvolvidas diretamente com os usuários. }\end{array}$ \\
\hline & Resolutividade & $\begin{array}{l}\text { Desenvolvimento de estratégias para garantir a resolução da maior parte dos problemas } \\
\text { dos indivíduos e comunidades no nível primário. Para tanto, o NASF deve atuar em face } \\
\text { dos determinantes sociais de saúde e na resolutividade clínica dos problemas complexos e } \\
\text { desafiadores que demandam suporte especializado. Considera as contribuições do } \\
\text { NASF-AB para a qualificação do cuidado e resolução dos problemas de saúde. }\end{array}$ \\
\hline & $\begin{array}{l}\text { Articulação com } \\
\text { a RAS }\end{array}$ & $\begin{array}{l}\text { Atuação para a garantia da integralidade do cuidado, com coordenação dos casos que } \\
\text { não podem ser resolvido na atenção primária. Para tanto, é necessária a existência da RAS } \\
\text { fortalecida e o conhecimento dos profissionais da APS sobre os pontos da rede, com canais } \\
\text { de comunicação e fluxos bem estabelecidos. Refere-se à atuação do } \\
\text { NASF na articulação entre as equipes de APS e os demais serviços de saúde, } \\
\text { com qualificação dos encaminhamentos. }\end{array}$ \\
\hline
\end{tabular}

EqSF: equipes de saúde da família; NASF-AB: Núcleo Ampliado de Saúde da Família e Atenção Básica; RAS: Rede de Atenção à Saúde. Fonte: elaborado com base em Starfield 2; Sanchez \& Ciconelli 25; Kringos 26; Campos et al. 27; Oliveira \& Campos 28; Ministério da Saúde 6,11. 
$\mathrm{O}$ atributo da integralidade considerou a necessidade de as equipes proverem ou garantirem os serviços que atendam às necessidades dos usuários e foi composto das seguintes dimensões: abrangências de ações, resolutividade e articulação com a rede de atenção. Esse atributo visa a identificar as contribuições do NASF para ampliação do cuidado na APS e as contribuições para a qualificação do cuidado.

Como fonte de informações, foram realizadas 44 entrevistas semiestruturadas com usuários que participaram de atividades com os apoiadores, como consultas individuais, visita domiciliar ou atividades coletivas, nos três meses anteriores ao período de realização do estudo.

Os usuários foram selecionados a partir de indicação dos enfermeiros e de agentes comunitários de saúde (ACS), que agendavam as entrevistas na unidade de saúde ou no domicílio do usuário. Os critérios de inclusão foram os seguintes: ter participado de alguma atividade do NASF nos três meses anteriores à coleta e ter disponibilidade de participar da pesquisa. Foram excluídas pessoas com dificuldades de comunicação verbal ou em estado de confusão mental. O número de entrevistas por município foi estabelecido pelo atendimento aos critérios de inclusão e exclusão no período de visita da equipe de pesquisa em cada localidade. A distribuição das entrevistas foi: Vitória da Conquista (16), Guanambi (7); Brumado (8), Poções (3); Barra do Choça (5); Belo Campo (5). Também foram utilizadas observações de campo para composição dos dados. As atividades observadas foram: atividades de grupo com usuários; visitas domiciliares; sala de espera; reuniões dos núcleos e entre equipes NASF e EqSF; rotina para agendamento e marcação de atividades. A fase de campo ocorreu entre março e dezembro de 2017.

As entrevistas foram gravadas em aparelho de áudio digital. Foram realizadas pelos dois primeiros autores deste artigo e por uma pesquisadora treinada. Para a condução das entrevistas, foi utilizado um roteiro que versava sobre a compreensão, importância e resolutividade das atividades desenvolvidas, acesso e tipos de ações realizadas, encaminhamentos a serviços especializados e satisfação do usuário. Para organização e categorização dos dados e informações, utilizou-se a técnica de Análise de Conteúdo Temática, proposta por Gibbs 29.

A pesquisa foi aprovada pelo Comitê de Ética em Pesquisa do Instituto Multidisciplinar em Saúde, Universidade Federal da Bahia (IMS-UFBA), parecer número 377.448. Todos os requisitos éticos foram observados, e os participantes assinaram o Termo de Consentimento Livre e Esclarecido.

\section{Resultados}

\section{Acesso}

A disponibilidade de ações e serviços do NASF mostrou-se restrita. Os resultados identificaram a realização de atividades de educação em saúde, atividade física, grupos terapêuticos, atendimentos individualizados e visitas domiciliares. Observou-se maior direcionamento para as ações grupais.

As ações disponibilizadas, tanto coletivas quanto individuais, foram insuficientes diante das demandas das comunidades. Evidenciaram-se baixa frequência na realização das atividades grupais e elevado tempo de espera para a marcação de consultas com os profissionais. Os usuários expressaram a necessidade de que as atividades coletivas fossem realizadas em maior frequência, uma vez que os grupos de atividade física acontecem, na maior parte das localidades, uma vez por semana, e os de educação em saúde são geralmente mensais ou esporádicos. Dificuldades para o agendamento de atendimentos individualizados e para as visitas domiciliares indicam limitações na disponibilidade. "Eu acho assim, ruim, né? Porque você espera muito. Mas, às vezes, também eu entendo que tem muitas pessoas" (usuário 44).

Sobre a acessibilidade, observou-se variedade de mecanismos para o usuário ter contato com o NASF. Prioritariamente, as formas de acesso da população às equipes de apoio foram por meio dos ACS. Com menor frequência, ocorrem encaminhamentos dos enfermeiros e médicos, bem como encaminhamentos por outros profissionais do núcleo e acesso direto ao apoiador. Os apoiadores também utilizaram as atividades coletivas como mecanismos de triagem e de seleção dos usuários para a atenção individualizada. 
"É a partir do grupo. Aí ela pergunta quem é que esteja necessitado, ai ela tem uma conversa, aí quando ela vê uma pessoa tá necessitando, então ela vai prosseguindo mais o andamento" (usuário 28).

A presença dos apoiadores nas comunidades facilitou o acesso a serviços antes disponíveis apenas na atenção secundária ou nos serviços privados. Além de diminuir o tempo de deslocamento dos usuários, essa presença também mitiga as questões financeiras que interferem no acesso, tanto referentes ao custo de deslocamento quanto à maior possibilidade de uso de serviços antes não disponíveis no nível primário.

A ampliação das visitas domiciliares foi outro fator que muito contribuiu para a melhoria da acessibilidade. Usuários com restrições de mobilidade passaram a ter contato com os profissionais por meio do atendimento domiciliar realizado pelos apoiadores. "Porque, tipo assim, visita o doente, né? Pra ver alguma coisa que precisa, né? Pra ajudar. (...) assim, a pessoa visitar um acamado, eu acho importante" (usuário 44).

Sobre a aceitabilidade do trabalho dos núcleos, os resultados foram controversos, com indicativos de satisfação e gratidão pela presença dos especialistas e, ao mesmo tempo, de frustrações de expectativas não cumpridas em face das demandas existentes. Os aspectos positivos da aceitabilidade referiram-se à possibilidade de acesso às especialidades e à postura acolhedora dos profissionais. "Que aqui mesmo, eles vieram várias vezes, não foi a troco de dinheiro, não foi a troco de nada. Foi a troco da bondade deles. Daquele amor. Que tem com o próximo” (usuário 20).

Diante do cenário de vulnerabilidade em que estão inseridos e das dificuldades para o acesso a outros níveis de atenção à saúde, os usuários valorizam a existência do NASF por vislumbrarem novas possibilidades de acesso. Todavia, a forma organizativa do trabalho dos núcleos, com priorização de atividades coletivas em detrimento dos atendimentos individualizados, gerou sentimento de frustração e de baixa aceitabilidade. Assim, os resultados revelaram resistências aos tipos de atividades desenvolvidas e, consequente, baixa adesão da população às atividades coletivas.

\section{Longitudinalidade}

A longitudinalidade do cuidado mostrou-se como atributo pouco valorizado pelos profissionais do NASF. O trabalho desenvolvido raramente é pautado na atenção continuada aos usuários. Foi revelada uma lógica organizativa do trabalho com avaliação inicial do usuário e encaminhamento para outros pontos da rede de atenção, quando se constatava a necessidade de seguimento. Nesse sentido, revela-se a não responsabilização do NASF pela continuidade do cuidado, mesmo diante de casos complexos e desafiadores. Faz o contato e pronto. "Aí, se for o caso, o menino passar em outro psicólogo, dá encaminhamento pra outro psicólogo. Mas, o NASF mesmo não acompanha” (usuário 22).

Os usuários salientaram a necessidade e a importância da continuidade da atenção pelos apoiadores. O conhecimento dos problemas do território e das condições de vida, assim como as vantagens do atendimento próximo ao domicílio, foram os principais fatores alegados na defesa do acompanhamento dos casos pelos núcleos.

Sobre o estabelecimento de vínculo entre profissionais dos núcleos e usuário, um achado relevante foi a capacidade de as ações coletivas contribuírem com o desenvolvimento de relações de confiança. Foi destacado que o conhecimento do profissional acerca das singularidades dos participantes dos grupos favorece o desenvolvimento de atividades mais efetivas e amplia a disposição dos usuários para seguir as orientações dos apoiadores.

"É importante que a gente cria mais amizade, a gente aconchega mais com as pessoas, a gente vê o cuidado das pessoas da equipe, né? (...) com o grupo lá dos idosos, né? E elas incentivam a gente a fazer as atividades, é muito bom" (usuário 34).

No entanto, a descontinuidade da atenção nos atendimentos individuais afetou como os usuários percebem o cuidado ofertado e abalam as relações de confiança. "A continuação, seria interessante, viu? Porque, você pega mais confiança, mais conhecimento com a pessoa” (usuário 24).

Ao realizar apenas encaminhamentos dos casos após avaliação inicial, ocorre a fragilização das relações de confiança da comunidade com os apoiadores, aspectos intrinsecamente relacionados com o vínculo. Como agravante, a frágil interação entre NASF e ESF também interferiu na manutenção do vínculo e do cuidado longitudinal no âmbito da APS. 
Ainda como elemento da dimensão vínculo, os resultados indicaram que as atividades educativas realizadas exerceram considerável influência na promoção da autonomia dos usuários. Foi evidenciado que o trabalho dos apoiadores nas comunidades resulta em elevação da consciência sanitária, transformações de hábitos de vida e incrementos nas ações de promoção da saúde e prevenção de agravos.

"Assim, têm pessoas mesmo, que já tão assim numa idade, eles não ligam mais (...) Muito pra essas coisas. Eu acho que já tem esse grupo que fala com a pessoa, a pessoa vai e (...) E termina fazendo essas atividades. Então, eu já vi muita pessoa de idade aqui, depois desse grupo, fazendo caminhada..." (usuário 08).

Mesmo diante da diminuta frequência das atividades coletivas e do seu caráter programático, os resultados sugerem maior predisposição dos participantes ao autocuidado. Tal disposição foi relacionada ao estabelecimento de vínculo entre profissionais e usuários, assim como ao desenvolvimento continuado dos grupos, ainda que com baixa frequência semanal. O vínculo demonstrou influência nas atitudes dos usuários para seguir as orientações dos profissionais e desenvolver corresponsabilização pela própria saúde.

\section{Integralidade}

O trabalho do NASF, embora revele alguns avanços, apresenta ainda consideráveis limitações em direção à integralidade. Sobre a abrangência das ações, evidenciou-se que a presença dos núcleos possibilitou o incremento de atividades e de serviços desenvolvidos na atenção primária. "Eu já participei da educação física, de palestra também, que é um grupo (...) Nutricionista, é muito bom, entendeu?" (usuário 23).

As atividades de caráter coletivo foram as que mais se ampliaram. Dentre essas, destacam-se os grupos educativos, terapêuticos e de atividade física. Também as visitas domiciliares foram ampliadas com a chegada dos apoiadores. Em menor proporção, foram incrementados atendimentos clínicos de profissionais com formação distinta dos integrantes da equipe mínima. "Porque antes, meu filho não passava por psicólogo, por nutricionista. Porque era muito difícil, sabe?” (usuário 17).

Embora tenham se destacado insatisfações com a forma de organização do trabalho e com as demandas não suficientemente atendidas, os resultados revelaram a importância dos núcleos para a ampliação do escopo das ações.

Sobre a dimensão resolutividade, foram identificados avanços e restrições do trabalho do NASF para a resolução dos problemas de saúde das comunidades. As ações coletivas emergiram como capazes de contribuir para a resolução de problemas. Os usuários destacaram melhorias nos estados de saúde dos participantes dos grupos educativos e terapêuticos. Foram referidos benefícios relacionados a sociabilidade e interação comunitária, perda de peso, maior disposição e remissão na agudização de condições crônicas. "É que eu sentia muita dor no corpo, dor nas pernas, dor nos braços, e depois que eu estou fazendo parte do grupo eu melhorei bastante" (usuário 06).

As equipes de apoio apresentaram maior facilidade para o acionamento e a articulação com as redes de proteção, no intuito de resolver problemas e necessidades que envolviam questões de vulnerabilidade econômica e social. "Ela [assistente social] veio aqui na minha casa (...) Através dela que eu consegui o medicamento. Eu precisei e consegui” (usuário 43).

Por sua vez, a resolutividade mostrou-se restrita para os casos complexos e que demandaram atendimento ou apoio clínico. A atuação pouco resolutiva foi associada à própria secundarização dos atendimentos clínicos, ao excesso de demanda, à baixa frequência dos grupos terapêuticos e ao cuidado não articulado entre núcleos e equipes apoiadas.

A articulação do NASF com a rede de atenção à saúde revelou-se inexpressiva. Os resultados evidenciaram não existir interação entre os apoiadores e os serviços secundários e terciários. Diante da necessidade de atendimento clínico em serviços de maior densidade tecnológica, realiza-se o encaminhamento dos profissionais de apoio para os médicos das EqSF, e são eles que procedem com o encaminhamento para os outros níveis de atenção. "Só encaminhou. Não fez nada aqui não. É tudo lá em cima, lá no setor. (...) Não foi ela [nutricionista], que encaminhou não. Quem me deu foi a doutora” (usuário 33).

A vivência dos usuários indica que a atuação do NASF pouco contribui para a coordenação do cuidado e a qualificação dos encaminhamentos. O fluxo estabelecido fundamentou-se em um processo exclusivamente burocrático, em decorrência de os encaminhamentos para os outros níveis serem atividades restritas aos médicos das EqSF. 


\section{Discussão}

O estudo demonstrou potencialidades e limitações na atuação do NASF em face dos atributos analisados. Apesar dos avanços observados, ainda persistem limitações no acesso dos usuários aos núcleos e na atuação longitudinal dos apoiadores, bem como fatores que comprometem a integralidade. É importante ressaltar que os resultados da avaliação devem ser interpretados e discutidos considerando as especificidades e nuances do trabalho dos núcleos. Embora sejam considerados integrantes da APS, os atributos utilizados não podem ser avaliados de igual maneira a uma EqSF, visto que os NASF não são serviços de acesso direto, tampouco são responsáveis pelo acompanhamento longitudinal de toda a população vinculada.

No atributo do acesso, foram evidenciadas importantes restrições na disponibilidade dos serviços. Embora existisse um leque de ações disponíveis, elas não eram realizadas de forma suficiente para atender às demandas da população. O estudo revelou reduzida oferta, tanto de ações individuais como de ações coletivas, com maior dificuldade para acesso às ações clínicas individualizadas.

Os usuários associaram a disponibilidade restrita ao número excessivo de equipes e pessoas apoiadas por cada núcleo. A sobrecarga dos profissionais dos núcleos foi também identificada em outros cenários 13,17. Cabe refletir que a sobrecarga em questão não é decorrente apenas do número de equipes apoiadas ser maior do que o preconizado. Os próprios parâmetros ministeriais podem ser indutores da limitação do acesso aos especialistas. A conformação de um NASF apoiar até 9 equipes de atenção básica (EqAB) ou EqSF 11 pressupõe um tipo de apoio que não é capaz de ofertar a retaguarda necessária aos usuários e equipes.

$\mathrm{Na}$ dimensão acessibilidade, o trabalho dos apoiadores demonstrou contribuir para a ampliação do acesso às ações e aos serviços na APS. Dois fatores favoreceram a acessibilidade dos usuários: a minimização das limitações de deslocamento e a maior oferta de serviços. As barreiras geográficas e financeiras muitas vezes se potencializam mutuamente e ampliam as dificuldades dos usuários no acesso aos serviços de saúde. Segundo Viegas et al. 30, a limitação do acesso advém da distância entre serviços e residências, mas também das dificuldades de transporte público para deslocamento. Nesse sentido, a ampliação das visitas domiciliares representou importante mecanismo de incremento do acesso, tanto por ampliar a disponibilidade, como por favorecer a acessibilidade.

Por sua vez, a indefinição dos mecanismos de acesso dos usuários aos profissionais de apoio revela fragilidades na forma organizativa do trabalho entre as equipes. $\mathrm{O}$ não estabelecimento de mecanismos regulares de contato com os núcleos também foi observado por Souza \& Medina ${ }^{17}$, que identificaram inexistência de critérios de encaminhamentos e informalidade dos fluxos. Por não ser um serviço de acesso direto, esses aspectos se constituíram em barreira organizativa dos serviços.

Ambíguas perspectivas marcaram a dimensão da aceitabilidade. O sentimento da gratidão pela existência dos núcleos coexistiu com a sensação de não alcance da atenção ou serviço de que necessitavam. De acordo com Fausto et al. 10, a avaliação de serviços baseada na experiência e satisfação dos usuários é complexa e sofre influência de diferentes valores sociais e culturais. Em contextos de restrição de acesso e onde os serviços são entendidos como favor e não como direito, o grau de satisfação tende a ser influenciado por sentimento de gratuidade. Diante das restrições da rede de atenção à saúde nos municípios estudados, especialmente no que tange à disponibilidade de serviços especializados, o imaginário dos usuários sobre a atenção ofertada pelo NASF pareceu se aproximar da ideia de caridade.

Sobre o atributo da longitudinalidade, os achados expressaram o diminuto envolvimento dos núcleos ampliados no desenvolvimento do cuidado de longa duração. Na avaliação dos usuários, o cuidado continuado por parte do NASF traria mais potência para APS em virtude desses profissionais conhecerem os problemas sociais e de saúde dos territórios. Assim, se apresentaria uma nova perspectiva de cuidados no SUS com a atuação de profissionais especialistas contextualizados com as realidades locais. Isso configura-se como abordagem inovadora, visto que, na maior parte das vezes, especialistas atuam focados em problemas específicos e desconectados do contexto de vida das populações.

O estudo revelou o distanciamento e a desresponsabilização dos núcleos em face da longitudinalidade, como se tal atributo não compusesse o seu leque de ações. Estudos internacionais $31,32 \mathrm{e}$ realizados no Brasil 20,33 apontam que avaliações positivas sobre a amplitude da APS perpassam pelo 
desenvolvimento da atenção ao longo do tempo e pelo conhecimento do profissional acerca dos problemas dos usuários. Por sua vez, situações de descontinuidade assistencial, como as evidenciadas na presente investigação, indicam o baixo envolvimento do NASF com o cuidado ao longo do tempo e com a integralidade. Ao restringir os atendimentos clínicos a avaliações e encaminhamentos para outros serviços, os profissionais dos núcleos inibem a potência do próprio trabalho e limitam as contribuições para a APS integral.

A desatenção com a longitudinalidade guardou relação com a ação desarticulada entre equipes de NASF e de saúde da família. É preconizado que a atuação dos núcleos ocorra de forma integrada e colaborativa com as EqSF, inclusive com a definição conjunta de projetos terapêuticos e o compartilhamento de responsabilidades 11. No entanto, o cuidado colaborativo entre os núcleos e as EqSF mostrou-se como uma prática ainda distante. Isso pode ser influenciado pelo pouco exercício da prática clínica por parte do NASF. Assim, as EqSF suportariam sozinhas a pressão assistencial que os apoiadores não vivenciam, por estarem protegidos pelas restrições de agenda em face do amplo leque de ações generalistas e coletivas 21 .

Enquanto a descontinuidade no acompanhamento clínico assistencial demostrou a fragilidade do NASF no que concerne ao atributo da longitudinalidade, as práticas de grupo revelaram que o trabalho dos apoiadores propicia o estabelecimento do vínculo e favorece a longitudinalidade das ações promocionais. Os resultados sugerem que os usuários percebem os grupos não só como espaço educativo e de promoção da saúde, mas, sobretudo, como evento social e espaço de convivência e descontração. Em estudo realizado em Minas Gerais, Brasil, também foi identificado que as atividades coletivas promovidas pelos núcleos propiciam a criação de vínculo afetivo e constitui-se como fator de satisfação dos usuários em relação às atividades desenvolvidas 14.

Um aspecto relevante evidenciado foi a capacidade dos grupos de produzirem resultados positivos sobre a saúde das populações. Os achados demostraram que os usuários participantes das atividades educativas tendem a adotar hábitos de vida mais saudáveis e cuidam melhor da própria saúde. Diante disso, é sugestivo que o trabalho dos núcleos tem grande potência para a promoção da saúde e para a atuação sobre os determinantes e condicionantes sociais.

No que concerne ao atributo da integralidade, as dimensões abrangência de ações e resolutividade demonstraram relevantes contribuições do trabalho do NASF para a APS. Por sua vez, a articulação com a rede de atenção evidenciou a frágil desenvoltura dos núcleos para a continuidade do cuidado em outros níveis de atenção. Nas duas dimensões em que se constataram avanços, o desempenho dos apoiadores se destacou especialmente pelas atividades coletivas desenvolvidas. Nesse sentido, os resultados desse atributo ratificam a relevância do trabalho dos núcleos na ampliação de ações promocionais, com capacidade de produzir resultados positivos sobre os determinantes sociais da saúde. Em contrapartida, a presença dos apoiadores pouco contribuiu para a amplitude e resolutividade dos atendimentos clínicos individuais.

Diversos estudos apontam a dicotomia entre atividades individuais e coletivas no desenvolvimento do trabalho dos núcleos e o difícil equilíbrio entre essas duas vertentes. Em muitas localidades, o trabalho dos núcleos tem se direcionado para as atividades coletivas 13,14,15, parecendo existir certa distorção sobre a organização do trabalho, como se os apoiadores estivessem impedidos de prestar cuidados curativos e reabilitadores 34 . Tesser \& Poli Neto 35 afirmam existir no âmbito da APS certa aversão à clínica do adoecido, e que os profissionais buscam predominantemente por atuação na promoção da saúde. Essa polarização induz ao distanciamento do suporte especializado pelos apoiadores, o que resulta em limitação do acesso da população diante de necessidades específicas. Nesse sentido, o atributo da integralidade é fragilizado e APS abrangente torna-se mais distante.

No que diz respeito à articulação com a RAS, os achados expressam que os núcleos se mantêm isolados no âmbito da APS. A presença dos profissionais especialistas não representou maior facilidade para os usuários terem acesso aos demais níveis de atenção, tampouco possibilitou a realização de encaminhamentos mais qualificados. Também nessa dimensão, o distanciamento das ações clínicas mostrou contribuir para a limitada atuação na articulação com a atenção especializada.

A coordenação do cuidado pela APS é apresentada na literatura internacional como elemento capaz de facilitar o acesso, ampliar a resolutividade e garantir o cuidado em tempo oportuno 26. No entanto, no cotidiano dos sistemas de saúde, constitui-se como elemento de difícil manejo. Nos países europeus, é o principal desafio em face da crescente demanda e complexidade dos serviços de 
saúde 36. As tentativas de integração desses sistemas de saúde interferem nos papéis tradicionais desempenhados por profissionais, prestadores e governos, o que desencadeia problemas de coordenação interprofissional, interorganizacional e intergovernamental 36. No Brasil, são persistentes as dificuldades de articulação da APS com a atenção especializada 20, e a coordenação do cuidado tornase difícil de se alcançar, dada a disponibilidade limitada de serviços especializados e a infraestrutura desigual de tecnologia da informação 9 .

Diante da avaliação das três dimensões estudadas, verificou-se que o trabalho dos núcleos ampliados apresenta avanços e limitações para a qualificação do cuidado aos usuários da APS. Os resultados da avaliação sugerem que a amplitude de atribuições a cargo do NASF interfere na atuação dos apoiadores e limita as contribuições para o alcance da APS abrangente. Somado a isso, as indefinições sobre o que se espera do trabalho e quais tipos de ações devem orientar as práticas de trabalho, se as clínico-assistenciais ou as coletivas-promocionais, favorecem o desenvolvimento de frustrações e descrédito pelos usuários. Nesse sentido, a atuação do NASF para o fortalecimento dos atributos da APS pode exigir estratégias de melhor definição do objeto de trabalho e delimitação mais específica das ações a serem desenvolvidas.

Não se pode deixar se considerar o contexto político, econômico e social que o Brasil vivencia, com rígidas políticas de austeridade iniciadas em 2016 que limitaram a expansão do gasto público e comprometem o orçamento do SUS 7,8. No âmbito da APS, a PNAB publicada em 2017 constituiuse em ponto de inflexão na direção da APS abrangente, com relativização da cobertura universal e segmentação do acesso 37 .

Especificamente sobre o NASF, a publicação da Portaria no 2.979/201938 configura-se em ameaça real à manutenção dos núcleos ampliados. Nessa portaria, são suprimidos os mecanismos de financiamento federal para sua implementação e seu custeio. Assim, muitos municípios enfrentarão dificuldades para a manutenção, o que pode significar a extinção do NASF em diversas localidades.

As lições aprendidas nesse estudo demonstram que o trabalho do NASF apresenta restrições no desenvolvimento dos atributos da APS abrangente. No entanto, os resultados também evidenciam o incremento de ações e serviços, bem como sugerem potencialidades pouco exploradas em um contexto de condições estrutural e organizacional restritivas. Espera-se que as reflexões apresentadas favoreçam o debate em torno do aprimoramento da função de apoio e fortalecimento da APS, em contraposição às medidas em curso de fragilização do SUS e de diminuição da proteção social.

\section{Colaboradores}

D. C. Moreira contribuiu com a coleta, análise e interpretação de dados, redação do manuscrito, revisão crítica do manuscrito e aprovação da versão final. J. P. Bispo Júnior contribui na concepção do estudo; coleta, análise e interpretação de dados, redação do manuscrito, revisão crítica do manuscrito e aprovação da versão final. A. A. Nery e J. P. Cardoso contribuíram na análise e interpretação de dados, revisão crítica do manuscrito e aprovação da versão final.

\section{Informações adicionais}

ORCID: Diane Costa Moreira (0000-0003-12637677); José Patrício Bispo Júnior (0000-0003-41559612); Adriana Alves Nery (0000-0002-1093-1437); Jefferson Paixão Cardoso (0000-0003-0128-5792).

\section{Agradecimentos}

À Fundação de Amparo à Pesquisa do Estado da Bahia (FAPESB), pelo apoio concedido por meio do Programa de Pesquisa para o SUS (PPSUS-BA), Edital 20/2013. 


\section{Referências}

1. Hone T, Macinko J, Millett C. Revisiting AlmaAta: what is the role of primary health care in achieving the Sustainable Development Goals? Lancet 2018; 392:1461-72.

2. Starfield B. Atenção primária: equilíbrio entre necessidades de saúde, serviços e tecnologia. Brasília: Organização das Nações Unidas para a Educação, a Ciência e a Cultura/Ministério da Saúde; 2002.

3. Giovanella L, Mendoza-Ruiz A, Pilar ACA, Rosa MC, Martins GB, Santos IS, et al. Sistema universal de saúde e cobertura universal: desvendando pressupostos e estratégias. Ciênc Saúde Colet 2018; 23:1763-76.

4. Cecilio LCO, Reis AAC. Apontamentos sobre os desafios (ainda) atuais da atenção básica à saúde. Cad Saúde Pública 2018; 34:e00056917.

5. Giovanella L. Atenção básica ou atenção primária à saúde? Cad Saúde Pública 2018; 34:e00029818.

6. Ministério da Saúde. Portaria no 2.436, de 21 de setembro de 2017. Aprova a Política Nacional de Atenção Básica, estabelecendo a revisão de diretrizes para a organização da Atenção Básica, no âmbito do Sistema Único de Saúde (SUS). Diário Oficial da União 2017; 22 set.

7. Castro MC, Massuda A, Almeida G, Massuda A, Menezes-Filho NA, Andrade MV, et al. Brazil's unified health system: the first 30 years and prospects for the future. Lancet 2019; 394:345-56.

8. Machado CV, Silva GAE. Political struggles for a universal health system in Brazil: successes and limits in the reduction of inequalities. Global Health 2019; 15 Suppl 1:77.

9. Macinko J, Harris MJ. Brazil's Family Health Strategy: delivering community-based primary care in a Universal Health System. N Engl J Med 2015; 372:2177-81.

10. Fausto MC, Bousquat A, Lima JG, Giovanella L, Almeida PF, Medonça, MH, et al. Evaluation of Brazilian Primary Health Care from the perspective of the users: accessible, continuous, and acceptable? J Ambul Care Manage 2017; 40 Suppl 2:S60-70.

11. Departamento de Atenção Básica, Secretaria de Atenção à Saúde, Ministério da Saúde. Núcleo de Apoio à Saúde da Família - volume 1: ferramentas para a gestão e para o trabalho cotidiano. Brasília: Ministério da Saúde; 2014. (Cadernos da Atenção Básica, 39).

12. Ministério da Saúde. Portaria no 99 , de 5 de fevereiro de 2020. Redefine registro das Equipes de Atenção Primária e Saúde Mental no Cadastro Nacional de Estabelecimentos de Saúde (CNES). Diário Oficial da União 2020; 11 fev.

13. Arce VAR, Teixeira CF. Práticas de saúde e modelo de atenção no âmbito do Núcleo de Apoio à Saúde da Família em Salvador (BA). Saúde Debate 2017; $41 \mathrm{n}$ spe 3:228-40.

14. Fernandes ETP, Souza MNL, Rodrigues SM. Práticas de grupo do Núcleo de Apoio à Saúde da Família: perspectiva do usuário. Physis (Rio J.) 2019; 29:e290115.
15. Klein AP, d'Oliveira AFPL. O "cabo de força" da assistência: concepção e prática de psicólogos sobre o Apoio Matricial no Núcleo de Apoio à Saúde da Família. Cad Saúde Pública 2017; 33:e00158815.

16. Bispo Júnior JP, Moreira DC. Cuidado colaborativo entre os Núcleos de Apoio à Saúde da Família e as equipes apoiadas. Physis (Rio J.) 2018; 28:e280310.

17. Souza TS, Medina MG. Nasf: fragmentação ou integração do trabalho em saúde na APS? Saúde Debate 2018; 42 n spe 2:145-58.

18. Aciole GG, Oliveira DKS. Percepções de usuários e profissionais da saúde da família sobre o Núcleo de Apoio à Saúde da Família. Saúde Debate 2017; 41:1090-101.

19. Yin RK. Estudo de caso: planejamento e métodos. 5a Ed. Porto Alegre: Bookman; 2015.

20. Bousquat A, Giovanella L, Fausto MCR, Medina MG, Martins CL, Almeida PF, et al. A atenção primária em regiões de saúde: política, estrutura e organização. Cad Saúde Pública 2019; 35 Suppl 2:e00099118.

21. Tesser CD. Núcleos de Apoio à Saúde da Família, seus potenciais e entraves: uma interpretação a partir da atenção primária à saúde. Interface (Botucatu) 2017; 21:565-78.

22. Vendruscolo C, Ferraz F, Tesser CD, Trindade LL. Núcleo Ampliado de Saúde da Família: espaço de interseção entre atenção primária e secundária. Texto \& Contexto Enferm 2019; 28:e20170560.

23. Ministério da Saúde. Sala de Gestão Estratégica e Participativa - SAGE. Saúde mais perto de você 2017. http:sage.saude.gov.br/\# (acessado em 22/Dez/2019).

24. Programa das Nações Unidas para o Desenvolvimento. Atlas do Desenvolvimento $\mathrm{Hu}-$ mano no Brasil (Atlas Brasil). http://atlasbra sil.org.br/2013/pt/home/ (acessado em 22/ Dez/2019)

25. Sanchez RM, Ciconelli RM. Conceitos de acesso à saúde. Rev Panam Salud Pública 2012; 31:260-8.

26. Kringos DS, Boerma WG, Hutchinson A, van der Zee J, Groenewegen PP. The breadth of primary care: a systematic literature review of its core dimensions. BMC Health Serv Res 2010; 10:65.

27. Campos GWS, Figueiredo MD, Pereira Júnior N, Castro CP. A aplicação da metodologia Paideia no apoio institucional, no apoio matricial e na clínica ampliada. Interface (Botucatu) 2014; 18:983-95.

28. Oliveira MM, Campos GWS. Matrix support and institutional support: analyzing their construction. Ciênc Saúde Colet 2015; 20:229-38.

29. Gibbs G. Análise de dados qualitativos. Porto Alegre: Artmed; 2009.

30. Viegas APB, Carmo RF, Luz ZMP. Fatores que influenciam o acesso aos serviços de saúde na visão de profissionais e usuários de uma unidade básica de referência. Saúde Soc 2015; 24:100-12. 
31. Adhikary G, Shawon MSR, Ali MW, Shamsuzzaman M, Ahmed S, Shackelford KA, et al. Factors influencing patients' satisfaction at different levels of health facilities in Bangladesh: Results from patient exit interviews. PLoS One 2018; 13:e0196643.

32. Doubova SV, Guanais FC, Pérez-Cuevas R, Canning D, Macinko J, Reich MR. Attributes of patient-centered primary care associated with the public perception of good healthcare quality in Brazil, Colombia, Mexico and El Salvador. Health Policy Plan 2016; 31:834-3.

33. Silva GS, Alves CRL. Avaliação do grau de implantação dos atributos da atenção primária à saúde como indicador da qualidade da assistência prestada às crianças. Cad Saúde Pública 2019; 35:e00095418.

34. Moreira DC, Soares DA, Castro CP, Bispo Júnior JP. Atuação do Núcleo de Apoio à Saúde da Família no fortalecimento da atenção primária: experiências dos agentes comunitários. Physis (Rio J.) 2019; 29:e290304.
35. Tesser CD, Poli Neto P. Atenção especializada ambulatorial no Sistema Único de Saúde: para superar um vazio. Ciênc Saúde Colet 2017; 22:941-51

36. Greer S, Wismar MJF. Introduction: strengthening governance amidst changing governance. In: Greer S, Wismar M, Figueras J, editors. Strengthening health system governance: better policies, stronger performance. London: McGraw-Hill; 2016. p. 3-26.

37. Morosini MVGC, Fonseca AF, Lima LD. Política Nacional de Atenção Básica 2017: retrocessos e riscos para o Sistema Único de Saúde. Saúde Debate 2018; 42:11-24.

38. Ministério da Saúde. Portaria no 2.979, de 12 de novembro de 2019. Institui o Programa Previne Brasil, que estabelece novo modelo de financiamento de custeio da Atenção Primária à Saúde no âmbito do Sistema Único de Saúde. Diário Oficial da União 2019; 13 nov. 
Abstract

The study aimed to evaluate the work by the Expanded Family Health and Basic Healthcare Centers (NASF-AB), from the users' perspective, based on the attributes of comprehensive primary healthcare (PHC). This was a qualitative multiple case study conducted in six municipalities (counties) in the state of Bahia, Brazil. The theoretical and methodological frame of reference was an evaluative matrix based on the following attributes: access, longitudinality, and comprehensiveness. The matrix also considered the assumption of Inter-Consultation Support and the guidelines of the Family Health Strategy. Data and information were obtained from 44 interviews with users of the NASF-AB and observation of the centers' activities and routine work in the health units. The study showed that users have limited access to the NASF-ABs' activities. The availability of clinical care was insufficient, access was facilitated by home visits, and the acceptability was jeopardized by frustrations from unmet expectations. Longitudinal care has not been prioritized in the centers' work, with limited involvement by supporters in developing continuing care. Group activities showed the potential for forming bonds. In the attribute of comprehensiveness, the presence of NASF-ABs helped increase activities and case-resolution capacity, but the linkage with the healthcare network was negligible. In conclusion, the work by the NASF-AB displayed limitations for the development of comprehensive PHC. The results also suggest potentialities with the capacity to strengthen primary care that have not been fully explored.

Primary Health Care; Patient Care Team; Health Evaluation; Heath Policy; Unified Health System

\section{Resumen}

El objetivo del estudio fue evaluar el trabajo de los Núcleos Ampliados de Salud de la Familia y Atención Básica (NASF-AB), desde la perspectiva de los usuarios(a), a partir de las características de la atención primaria en salud (APS) integral. Estudio de evaluación cualitativa del tipo casos múltiples, desarrollado en seis municipios del estado de Bahía. Se utilizó como referencia teórica metodológica una matriz evaluativa, basada en los atributos: acceso, longitudinalidad e integralidad. La matriz también consideró los presupuestos del Apoyo Matricial y las directrices de la Estrategia Salud de la Familia. Las fuentes de datos e información fueron: 44 entrevistas con usuarios del NASF-AB y observación de las actividades de los núcleos y de las rutinas de las unidades de salud. Se evidenció que los usuarios tienen un acceso restringido a las actividades de los núcleos. La disponibilidad de la atención clínica se mostró insuficiente, la accesibilidad fue facilitada por la realización de visitas domiciliarias y la aceptabilidad se vio afectada por frustraciones, debidas a expectativas no cumplidas. La longitudinalidad del cuidado no ha sido priorizada en el trabajo de los núcleos, con una frágil implicación de quienes apoyan en el desarrollo del cuidado continuo. Las actividades grupales demostraron potencialidad para la creación de vínculos. En el atributo de la integralidad se identificó que la presencia de los núcleos contribuyó al incremento de acciones y la resolutividad, no obstante, la coordinación con la red de atención fue inexpresiva. Se concluye que el trabajo del NASF-AB presentó restricciones para el desarrollo de una APS integral. Los resultados también sugieren potencialidades con capacidad de fortalecer los cuidados primarios que se han investigado poco.

Atención Primaria en Salud; Grupo de Atención al Paciente; Evaluación en Salud; Política de Salud; Sistema Único de Salud
Recebido em 19/Fev/2020

Versão final reapresentada em 01/Mai/2020

Aprovado em 09/Jun/2020 